\title{
Japanese Dictionary for Sentiment Analysis of Counselling Text
}

Japanese Sentiment Dictionary

\author{
Masataka, Nakayama
}

Kokoro Research Center, Kyoto University, nakayama.masataka.4v [at] kyoto-u.ac.jp

Chihiro, Hatanaka

Kokoro Research Center, Kyoto University, hatanaka.chihiro.3e [at] kyoto-u.ac.jp

Hisae, Konakawa

Kokoro Research Center, Kyoto University, konakawa.hisae.8e [at] kyoto-u.ac.jp

Yuka, Suzuki

Kokoro Research Center, Kyoto University, suzuki.yuka.4n [at] kyoto-u.ac.jp

Alethea H. Q. Koh

Graduate School of Human and Environmental Studies, Kyoto University, alethea.koh.33z [at] st.kyotou.ac.jp

Yasushi, Sugihara

General Student Support Center, Kyoto University, sugihara.yasushi.5x [at] kyoto-u.ac.jp

\section{Toshio, Kawai}

Kokoro Research Center, Kyoto University, kawai.toshio.6c [at] kyoto-u.ac.jp

Chat-based counselling has become increasingly popular in the era of telecommunication. The need for accessible therapy has been exacerbated by the COVID-19 pandemic. Given its text-based nature, chat-based counselling provides an opportunity for machine-based analysis. It even has the potential to provide machine-based counselling services. However, the informational resources for machine-based analysis and interaction are rather scarce especially in a Japanese-language context. We created a Japanese dictionary for sentiment analysis, using a technique via machine-based text analysis, tailored for counselling related text. It includes 2389 words that were frequently used in chat-based counselling corpora. The following attributes were included for each word: (1) valence rating by the general public, (2) valence rating by clinical psychologists, (3) emotionality, and (4) body-relatedness.

CCS CONCEPTS • Psychology $•$ Heuristic evaluations $\bullet$ Language resources

Additional Keywords and Phrases: sentiment analysis, chat-based counseling, dictionary, clinical psychology ACM Reference Format: Masataka, Nakayama, Chihiro, Hatanaka, Hisae, Konakawa, Yuka, Suzuki, Alethea H. Q. Koh, Yasushi, Sugihara, and Toshio, Kawai. 2021. Japanese Dictionary for Sentiment Analysis of Counselling Text. In Proceedings of the 9th 
International Conference on Human-Agent Interaction (HAI '21), November 09-11, 2021, Virtual Event, Japan. ACM, New York, NY, USA, 5 pages. https://doi.org/10.1145/3472307.3484663

\section{INTRODUCTION}

Chat-based counselling has become increasingly popular in the era of telecommunication. The need for accessible therapy has been exacerbated by the COVID-19 pandemic. Given its text-based nature, chat-based counselling provides an opportunity for machine-based analysis [1]. It even has the potential to provide machine-assisted and machine-based counselling services. However, the informational resources for machinebased analysis are rather scarce especially in a Japanese-language context. One important technique of machine-based text analysis is sentiment analysis. Sentiment analysis allows one to approximate the writer's sentiment. In a clinical context, sentiment analysis, for example, can provide offline and/or online counselling assistance while adjusting the emotional valence of the therapists' message by estimating the clients' emotion (we return this point in "4.1 Application"). To enable sentiment analysis for Japanese counselling related text, we created a Japanese dictionary for sentiment analysis tailored for counselling related text.

\subsection{Sentiment Analysis}

Sentiment analysis is a technique to estimate emotional valence (i.e., sentiment) from text. The estimated valence is unidimensional and is either binary or continuous, typically consisting of a positive end represented by +1 and a negative end represented by -1 . The typical target is the writer's sentiment at $n$-gram, sentence, paragraph and other higher levels of units. The sentiment is estimated by using a dictionary where each word is registered with a valence. Dictionary-based approaches have been successful in various domains $[15,16]$. In clinical psychological contexts, emotion is an important variable. For example, a therapist's empathetic concern for a client is predictive of positive therapeutic outcome [6] and sentiment analysis has the potential to objectively and automatically evaluate such empathy. In addition, some clinical populations are characterized with disorders in emotion (e.g., major depressive disorder, persistent depressive disorder, generalized anxiety disorder, and affective disorder [2]) and sentiment analysis might be able to support assessment of such populations. Despite the potential utility of sentiment analysis in clinical contexts, available Japanese sentiment dictionaries $[8,10,11,17]$ are for general purposes and are not tailored to the clinical context.

\subsection{The current study}

In the current study, we constructed a sentiment dictionary that is tailored to the clinical context. We attempted to (1) maximize the coverage of clinically-relevant words and (2) include clinically-relevant attributes for each word. To maximize the coverage, we counted the frequency of words in large corpora of chat-based counselling sessions (see below) and selected high frequency words for the dictionary. For each word, valence was rated by a group of raters according to the scale mentioned above. We additionally included emotionality and bodyrelatedness as clinically-relevant attributes. We also included rater-related attributes, namely gender and expertise in clinical psychology.

Emotionality is a binary variable that indicates whether the word refers to an emotion (e.g., "happy") or not (e.g., "success"). This variable is important because differentiating subjective feelings from factual descriptions is important in a clinical context. Emotion is a subjective feeling or appraisal of external events and it should be differentiated from the factual evaluation of the contents of the event itself. For example, the loss of a loved one 
is a negative event that is likely to elicit the negative emotion of grief. However, this feeling towards the event is also likely to change as time passes (e.g., finding meaning in the event that may lead to positive appraisal; $[7,14])$. It is, therefore, clinically important to differentiate objective/normative evaluation of the event from the subjective experience of it. The emotionality attribute would allow the identification of subjective feelings. Emotionality was determined by ratings by clinical psychologists.

Body-relatedness is an important attribute because interoception plays an important role in emotion perception and mental health [3]. To this end, bodily states rather than emotions may be referred to during counselling. Patients with alexithymia have difficulties in subjectively feeling or labeling body states [13]. Somatization (i.e., talking about the body states instead of emotion) is more prevalent in East Asian cultural contexts than Western contexts [4]. For example, a "bad feeling" could be felt and expressed as a "headache". Importantly, somatization invited more sympathy in an East Asian context than a Western context [4]. Thus, a filter for bodyrelated words is clinically important, especially in the Japanese cultural context. Body-relatedness was determined by ratings by clinical psychologists.

Since we measured valence for each word by subjective ratings, rater-related attributes may or may not systematically influence the ratings by reflecting their different interpretations of the words. We separated ratings by participants from the general public (i.e., workers from a crowdsourcing site) and those by clinical psychologists. We also reported ratings separated by rater gender as well as an integrated version. As shown below, the ratings were highly correlated to each other but we additionally report examples of words with deviated ratings.

\section{METHOD}

We created the Japanese sentiment dictionary in three steps. In the first step, we selected candidate words for the dictionary from chat-based counselling corpora. In the second step, we conducted an online survey to collect subjective ratings from the general public for each word. In the third step, we collected subjective ratings from clinical psychologists for each word and ratings for emotionality and body-relatedness.

\subsection{Selection of Candidate Words.}

\subsubsection{Chat-based Counselling Corpora.}

Chat-based counselling services were provided to the Japanese public in March 2018 and in March 2019 by the Ministry of Health, Labour and Welfare. Before receiving the service, the receivers agreed on the use of the text obtained during counselling (i.e., counselling text) for research. The anonymized texts were provided by the counseling service provider to our research group. The analysis of the text was approved by a local ethics committee.

\subsubsection{Selection of Candidate Words.}

Each corpus was parsed into words by [9]. The corpus from 2018 consisted of 9495 types and 740363 tokens of words. The corpus from 2019 consisted of 4147 types and 247512 tokens of words. The total frequency of each word was counted and words with total token frequency of 20 or more were selected. When counting frequency, homographs were aggregated into one entry because human raters would not be able to distinguish the meaning of the word without context. Frequent but relatively meaningless or uninformative words were also 
excluded by the first author. Typical excluded words were words such as a part of a URL (e.g., "http") that was referred to by the therapist for information sharing purposes. There remained 2406 types of words.

\subsection{Online Survey for Human Subjective Ratings.}

\subsubsection{Participants.}

A total of 2088 participants were recruited from a crowd sourcing platform, Lancers (https://www.lancers.jp). The survey was separated into five runs and participants were allowed to participate multiple times across different runs and within the same run. Participation for each person was limited to 2 to 5 times per run, depending on the total number of recruited participants in each run. Participants received 50 JPY for each participation, which took about three minutes. All participants gave informed consent and the procedure of the online survey was approved by a local ethics committee.

\subsubsection{Materials.}

In addition to the selected 2406 words, 15 emotion-related words were included in the survey for exploratory purposes. Nonsense words were also used to check the attention of the participants who were asked to respond to nonsense words with "don't know". The data collection was separated into five runs. In each run, recruitment was terminated respectively when a total of $50,50,1000,500$, and 461 participants started the survey. The selection/presentation probabilities for each word were adjusted in each run to balance the number of ratings per word. Those words that received numerous "don't know" ratings were omitted from the survey after some runs were conducted, otherwise it would require more participants to balance the number of ratings for the words. The final set consisted of 2389 words.

\subsubsection{Procedure.}

Participants were instructed to rate the valence of individually presented words. A five-point Likert scale was used where 1 represented "negative" and 5 represented "positive". They were also asked to select the option "don't know" if they did not know the presented word. In a single participation, participants rated 74 words and two nonsense words that were pseudo-randomly selected, such that words were not repeated in each participation. At the end of the participation, participants were asked to provide their gender (male, female, or other) and age.

\subsubsection{Data Cleaning.}

Data were cleaned with the following criteria. Data were deleted in a trial-wise manner (1) if the reaction time to the trial was shorter than $400 \mathrm{~ms}$ or longer than $10000 \mathrm{~ms}$, or (2) if the response was "don't know". Data were deleted in a participation-wise manner (1) if $10 \%$ or more trials were deleted because of too short or too long reaction time, or (2) if the participant failed to pass the attention check (i.e., failing to select "don't know" for at least one nonsense word). On average 48.22 (range: $24-74$ ) participants rated each word.

\subsection{Human Subjective Rating by Clinical psychologists.}

To compare the ratings by the general public and by clinical psychologists, a group of clinical psychologists rated the valence of the words. Emotionality and body-relatedness were also judged by the clinical psychologists. 


\subsubsection{Raters.}

Eight clinical psychologists rated the valence, emotionality, and body-relatedness of the words. Seven were graduate students who majored in clinical psychology and one was a postdoctoral clinical psychology researcher (i.e., third author). Four rated all words for all three attributes and four rated a portion of the words for valence only.

\subsubsection{Procedure.}

Valence was rated with the same scale as the online survey. Emotionality was rated with a 3-point scale (0: "word that is not used to express emotion.", 1: "word that may or may not be used to express emotion. In other words, it possibly expresses emotion.", 2: "word that is [in most cases] used to express emotion"). Bodyrelatedness was rated with three categories (0: "a word that is unrelated to body parts or states.", 1: "a word that refers to body parts.", 2: "a word that is related to physical symptoms and/or bodily responses [it is not necessarily a negative word].").

For simplicity, emotionality and body-relatedness were aggregated and converted into binary variables. If $50 \%$ or more raters rated the emotionality of a given word as 1 or 2, then emotionality for the word was coded as 1 and otherwise as 0 . If at least one rater rated the body-relatedness of a given word as 1 or 2 , then bodyrelatedness for the word was coded as 1 and otherwise 0 . Original data is available upon request. Of all the words, $13.3 \%$ were emotional and $17.8 \%$ were body-related. Examples of emotional words are “憧れる /longing”, “羡ましい/envy”, and “可哀想/pity”. Examples of body-related words are “痛み/pain”, “震え/shudder', and “不眠症/insomnia”.

\section{PROPERTIES OF THE DICTIONARY}

The constructed dictionary is available at https://osf.io/fe7z5/. This section reports descriptive statistics for variants of valence, emotionality, and body-relatedness.

\subsection{Valence.}

Different variants of valence were estimated and compared (Figure 1).

\subsubsection{Estimation Methods.}

General_all was estimated by averaging all data from the general public. General_female was estimated by averaging the data from female raters from the general public. General_male was estimated by averaging the data from male raters from the general public. General_gender_weighted was estimated by averaging General_female and General_male. Unlike General_all, General_gender_weighted balances the weights of female and male raters. Raters who reported their gender as "other" were few and their data were included in General_all but not in General_gender_weighted. Clinical_psychologists was estimated by averaging all data from clinical psychologists.

\subsubsection{Means and SDs.}

The diagonal of Figure 1 plots the distribution of valence with each estimation method. Mean (SD) was 2.99 (0.786) for General_all, 2.99 (0.794) for General_female, 3.01 (0.827) for General_male, 2.98 (0.771) for General_gender_weighted, and 2.91 (0.684) for Clinical_psychologists. 

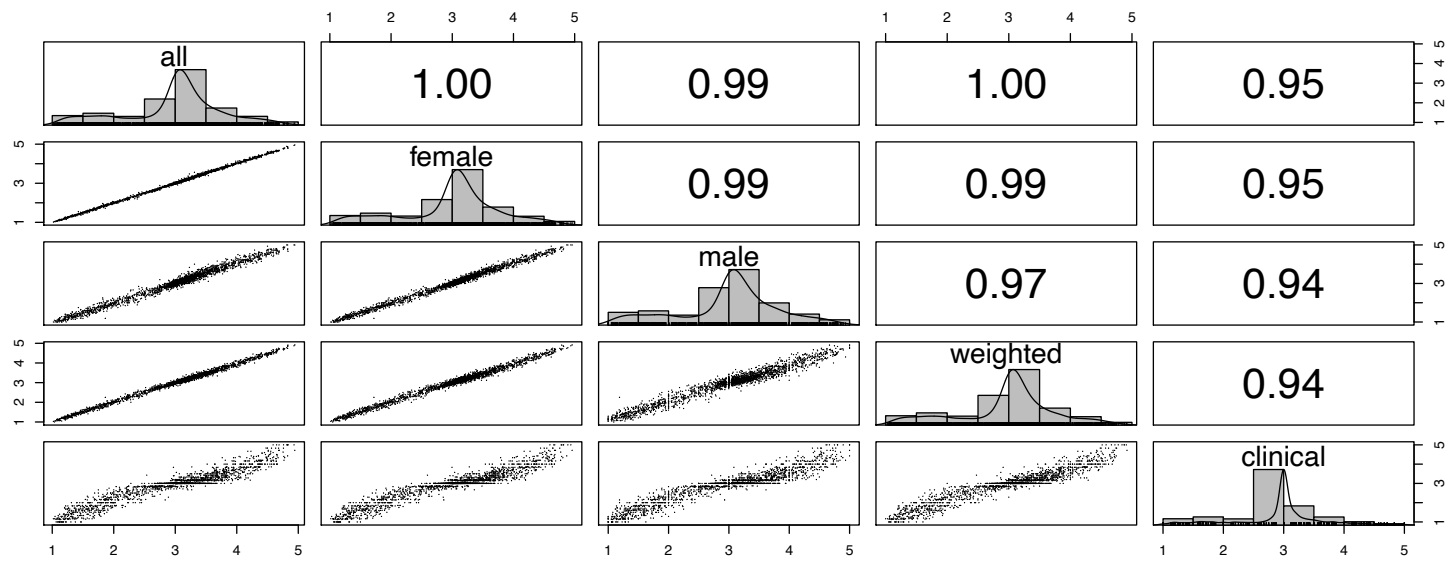

Figure 1: Distributions and correlations of valence estimates. All: general_all, female: general_female, male: general_male, weighted: general_gender_weighted, clinical: clinical psychologists.

\subsubsection{Correlations.}

Correlations among different valence estimates are displayed in Figure 1. The upper diagonal displays correlation coefficients and the lower diagonal displays scatterplots. Different estimates were highly correlated to each other ( $r s>.93)$, indicating high reliability of the estimates.

To demonstrate the potential usefulness of each specific estimate, we show some examples where different estimates diverged. Potential gender difference for valence was observed in some words. おかあさん (mother) was interpreted more positively by males (3.67) than females (3.11). Similarly, 姑 (mother-in-law) was interpreted less negatively by males (2.88) than females (2.33) and 父親 (father) was interpreted more positively by females (3.62) than males (3.11). Family relationship is a likely topic in the counseling context and finergrained estimates of valence would be useful.

Potential differences between the general public (general_all) and clinical psychologists were also observed in some words. 吐き出す (vent/vomit) was interpreted less negatively by clinical psychologists (2.71) than the general public (2.10), 闘病 (fight against disease) was interpreted less negatively by clinical psychologists (2.83) than general public (2.04), and スタート (start) was interpreted less positively by clinical psychologists (3.00) than general public (3.80). In clinical contexts, some actions, states and events might have more neutral and/or ambivalent (psychological) meaning diverging from normative meaning. For the above examples, vomiting something (including metaphorically) typically implies illness or aggression but it can also express catharsis. Disease may be negative but fighting against it may be psychologically positive. Lastly, the 'start' of something can result in bad as well as good consequence.

\subsubsection{Relationship to Emotionality.}

Figure 2 displays the distribution of the valence of emotional and non-emotional words. Not surprisingly, emotional words showed two peaks at the positive and negative ends, whereas non-emotional words peaked only once at neutral. 


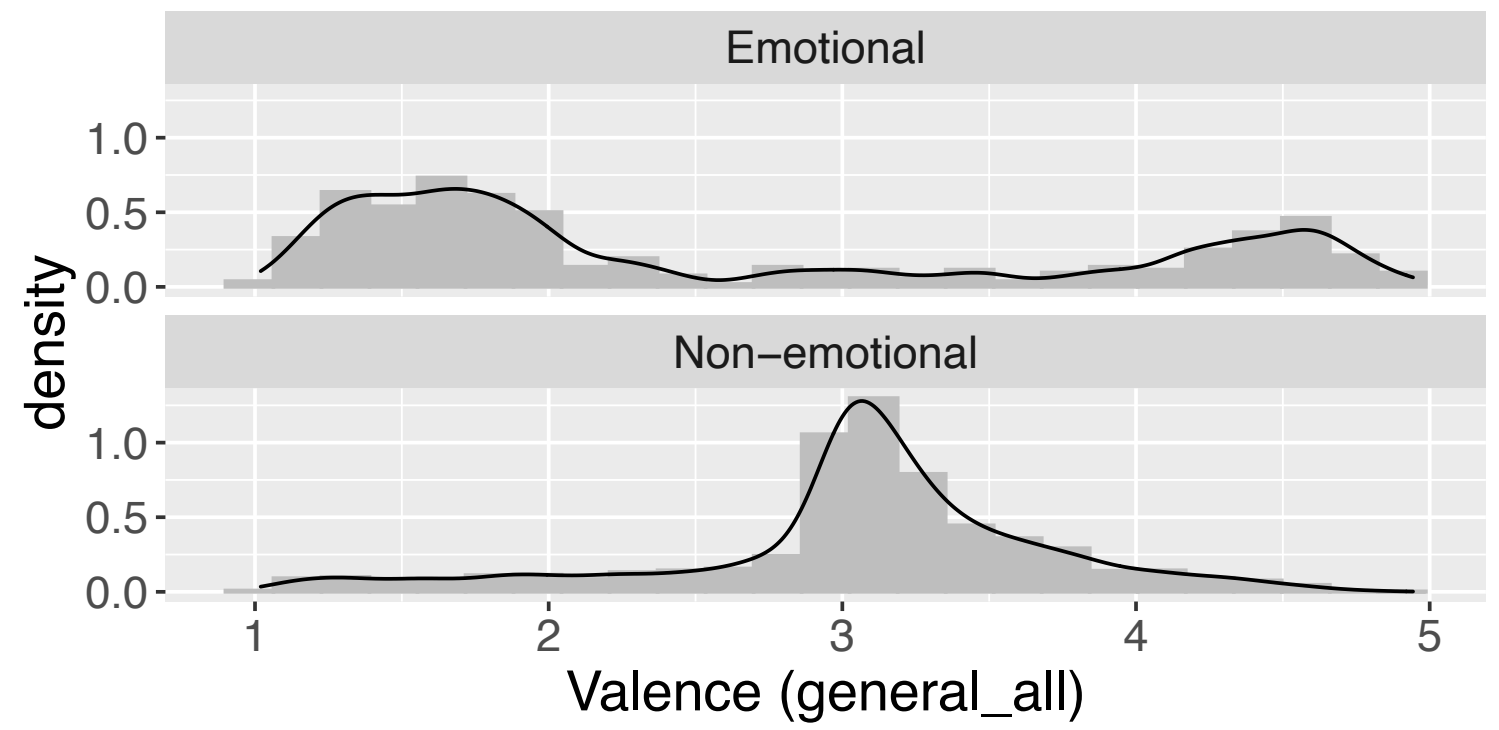

Figure 2: Distribution of valence (general_all) of emotional and non-emotional words.

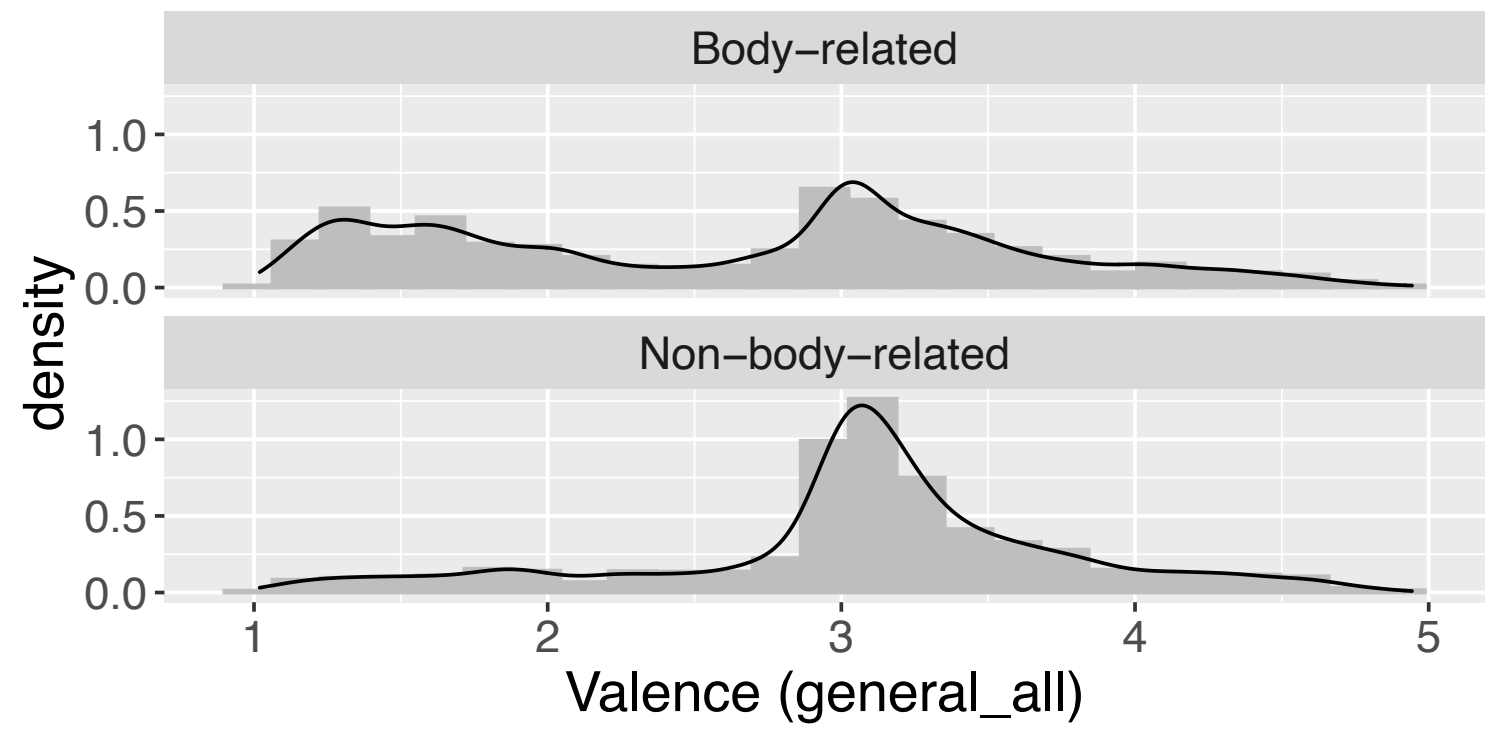

Figure 3: Distribution of valence (general_all) of body-related and non-body-related words.

\subsubsection{Relationship to Body-relatedness.}

Figure 3 displays the distribution of the valence of body-related and non-body-related words. Body-related words peaked twice at negative and neutral, whereas non-body-related words peaked only once at neutral. The negative peak may reflect somatization of negative affective states, while the neutral peak may reflect body parts. 


\section{DISCUSSION}

We created a Japanese dictionary for sentiment analysis that is tailored for counselling text. It includes 2389 words that were frequently used in chat-based counselling corpora. The following attributes were included for each word: (1) valence rating by the general public, (2) valence rating by clinical psychologists, (3) emotionality, and (4) body-relatedness. The next section discusses potential applications of this dictionary.

\subsection{Application.}

A potential application of the dictionary is to analyze and/or monitor temporal dynamics of sentiment in chatbased counselling. During counselling, the emotion felt by the client is not necessarily stable and changes in emotionality may reflect opportunities for therapeutic functions (e.g., [5]). Monitoring the sentiment with high time resolution (e.g., human ratings for each message in chat-based counselling) is impractical especially with large corpora. Dictionary-based sentiment analysis allows for the monitoring and/or analysis of the temporal dynamic of the sentiment in a more feasible way. To demonstrate its potential application, we [12] analyzed a chat-based counselling corpus with the current dictionary. Message-level sentiment was estimated and temporal patterns of the sentiment were analyzed by using latent growth class analysis. The analysis identified five classes of temporal patterns of sentiment. Of these classes, one class was associated with better session quality, and was characterized with (1) close alignment of sentiment between the counselor and client and (2) temporary negative shift in sentiment. As this example demonstrates, our sentiment dictionary provides a useful tool to analyze text from chat-based counseling. This analysis suggests a potentially useful application for offline and/or online assistance systems using our dictionary. If the system provides the emotional valence of both client's and therapist's message, it assists (non-expert) therapists in the tailoring of their message according to the client's emotionality, reducing discrepancy due to the enhanced emotional accuracy provided by the dictionary.

\section{ACKNOWLEDGMENTS}

This work was supported by JSPS KAKENHI Grant Numbers 19K21815 and 21H00948, and by SPIRITS 2020 of Kyoto University.

\section{REFERENCES}

[1] Althoff, T. et al. 2016. Large-scale Analysis of Counseling Conversations: An Application of Natural Language Processing to Mental Health. Transactions of the Association for Computational Linguistics. 4, (2016), 463-476. DOI:https://doi.org/10.1162/tacl_a_00111.

[2] American Psychiatric Association 2013. Diagnostic and Statistical Manual of Mental Disorders. American Psychiatric Association.

[3] Barrett, L.F. and Simmons, W.K. 2015. Interoceptive predictions in the brain. Nature Reviews Neuroscience. 16, 7 (2015), 419429. DOI:https://doi.org/10.1038/nrn3950.

[4] Choi, E. et al. 2016. The effectiveness of somatization in communicating distress in Korean and American cultural contexts. Frontiers in Psychology. 7, MAR (2016), 1-17. DOI:https://doi.org/10.3389/fpsyg.2016.00383.

[5] Cooper, S.H. 2007. Alexander's Corrective Emotional Experience: an Objectivist Turn in Psychoanalytic Authority and Technique. The Psychoanalytic Quarterly. 76, 4 (Oct. 2007), 1085-1102. DOI:https://doi.org/10.1002/j.2167-4086.2007.tb00294.x.

[6] Elliott, R. et al. 2011. Empathy. Psychotherapy. 48, 1 (2011), 43-49. DOI:https://doi.org/10.1037/a0022187.

[7] Heine, S.J. et al. 2006. Meaning Maintenance Model. Personality and Social Psychology Review. 10, 2 (2006), 88-110. 
Higashiyama, M. et al. 2008. Learning Sentiment of Nouns from Selectional Preferences of Verbs and Adjectives. Proceedings of the 14th Annual Meeting of the Association for Natural Language Processing (2008), 584-587.

[9] Ishida, M. 2017. RMeCab: Interface to MeCab (R package version 0.99997).

[10] Liew, K. et al. 2021. Tuning and validation of the JIWC Japanese emotion lexicon. Society for Affective Science Annual Conference (online, 2021).

[11] Miura, A. et al. 2015. Expression of negative emotional responses to the 2011 Great East Japan Earthquake: Analysis of big data from social media [in Japanese]. Japanese Journal of Psychology. 86, 2 (2015), 102-111.

[12] Nakayama, M. et al. 2021. Patterns of temporal affect dynamics in chat-based counselling. Society for Affective Science Annual Conference (2021).

[13] Panayiotou, G. et al. 2015. Do alexithymic individuals avoid their feelings? Experiential avoidance mediates the association between alexithymia, psychosomatic, and depressive symptoms in a community and a clinical sample. Comprehensive Psychiatry. 56, (2015), 206-216. DOI:https://doi.org/10.1016/j.comppsych.2014.09.006.

[14] Park, C.L. 2010. Making Sense of the Meaning Literature: An Integrative Review of Meaning Making and Its Effects on Adjustment to Stressful Life Events. Psychological Bulletin. 136, 2 (2010), 257-301. DOI:https://doi.org/10.1037/a0018301.

[15] Reagan, A.J. et al. 2017. Sentiment analysis methods for understanding large-scale texts: a case for using continuum-scored words and word shift graphs. EPJ Data Science. 6, 1 (2017). DOI:https://doi.org/10.1140/epjds/s13688-017-0121-9.

[16] Serrano-Guerrero, J. et al. 2015. Sentiment analysis: A review and comparative analysis of web services. Information Sciences. 311, (2015), 18-38. DOI:https://doi.org/10.1016/j.ins.2015.03.040.

[17] Takamura, H. et al. 2005. Extracting semantic orientations of words using spin model. ACL-05 - 43rd Annual Meeting of the Association for Computational Linguistics, Proceedings of the Conference. 47, 2 (2005), 133-140.

DOI:https://doi.org/10.3115/1219840.1219857. 